\title{
Validación de un sistema de monitoreo remoto para el control de estrés térmico en pollo de carne
}

\section{Validation of a remote monitoring system for the control of thermal stress in meat chicken}

Pablo Alfredo Rituay Trujillo1, Marcelo Eduardo de Oliveira2, Jonathan Alberto Campos Trigoso3,

Adriano Rogério Bruno Tech4

\section{RESUMEN}

Según la Asociación Brasilera de Proteína Animal (ABPA) en el mundo existe cerca de 19,6 millones de pollos de carne, el Brasil exporta a más de 150 mercados internacionales abasteciendo con aproximadamente 4 millones de toneladas anuales, esto representa un tercio del total producido en el país. La producción de pollos de carne brasilera actualmente afronta diversos desafíos en la búsqueda del bienestar animal y de su rentabilidad, el clima tropical con temperatura y niveles de irradiación solar elevadas en gran parte del año representan las mayores dificultades que provocan estrés térmico, por eso, es de extrema importancia que las instalaciones productivas aseguren la temperatura ideal. El objetivo de la investigación fue validar un sistema de monitoreo utilizando una red inalámbrica para el control térmico aplicado en un aviario experimental y verificar el desempeño operacional del sistema, el almacenamiento de los datos y el acceso de los mismos en tiempo real por medio de la web. Se analizaron los datos colectados durante 28 días, obteniendo resultados que validaron el sistema, comprobando que es posible medir la temperatura ideal para controlar la misma dentro del aviario con el objetivo de evitar el estrés térmico de las aves.

Palabras clave: Sensores; Actuadores; Sistema micro controlado; e-Science.

\begin{abstract}
According to the Brazilian Association of Animal Protein (ABPA) in the world there are about 19.6 million chickens of meat, Brazil exports to more than 150 international markets supplying approximately 4 million tons per year, this represents one third of the total produced in the country. Brazilian chicken production currently faces several challenges in the search for animal welfare and its profitability, the tropical climate with temperature and high levels of solar radiation in much of the year represent the greatest difficulties that cause thermal stress, therefore, It is extremely important that the production facilities ensure the ideal temperature. The objective of the research was to validate a monitoring system using a wireless network for the thermal control applied in an experimental aviary and verify the operational performance of the system, the storage of the data and their access in real time by means of the Web. We analyzed the data collected during 28 days, obtaining results that validated the system, verifying that it is possible to measure the ideal temperature to control it inside the aviary with the objective of avoiding the thermal stress of the birds.
\end{abstract}

Keywords: Actuators; Micro-controlled system; e-Science.

\footnotetext{
Alumno de la Escuela Profesional de Ingeniería en Agronegocios de la Universidad Nacional Toribio Rodríguez de Mendoza de Amazonas. Email: pablo.rituay@untrm.edu.pe

2Estudiante del Programa Doctoral de Ingeniería Ambiental en la Escuela de Ingeniería de São Carlos de la Universidad de São, Brasil. Email: meoli@usp.br ${ }^{3}$ Docente de la Escuela Profesional de Ingeniería en Agronegocios Universidad Nacional Toribio Rodríguez de Mendoza de Amazonas. Email: Jonathan.campos@untrm.edu.pe

${ }^{4}$ Docente de la Facultad de Zootecnia e Ingeniería de Alimentos de la Universidad de São, Brasil. Email: adriano.tech@usp.br
} 


\section{INTRODUCCIÓN}

En Brasil la producción de pollos de carne ha mostrado gran crecimiento en los últimos años, gracias al uso de nuevos métodos y tecnologías que permiten el control de temperatura del aire para lograr un ambiente ideal asegurando el bienestar animal. Sin embargo, se siguen enfrentando desafíos por factores ambientales. Considerando que el Brasil posee una gran diversidad climática y la temperatura e irradiación son elevadas en casi todo el año que generan estrés térmico en las aves, por lo que las variables del clima en una región dictan los niveles necesarios de control térmico en los ambientes de producción (Abreu y Abreu, 2004).

Según (Bridi, 2010), las aves son animales clasificadas como homeotérmicos, pues tienen la capacidad de regular el calor interno; siempre y cuando la temperatura del ambiente se encuentre dentro del parámetro óptimo de confort térmico (Abreu y Abreu, 2011).

Los altos niveles de temperatura del aire afectan directamente la respuesta de las aves al estrés térmico. Según Silva et al. (2007), cuando los pollos de carne están expuestos a temperaturas extremas externas (mayores a $35^{\circ} \mathrm{C}$ ) provocan hipertermia teniendo como desenlace la muerte del animal. Han, et al., 2010) experimentaron a una temperatura de 35 ${ }^{\circ} \mathrm{C}$ en aves de cuatro semanas de edad por 3 horas / día y observaron síntomas claros de estrés térmico

La industria avícola brasilera enfrenta los problemas de producción en zonas de altas temperaturas ambientales, con el desarrollo de instalaciones adaptadas de tecnologías adecuadas (Oliveira, et al., 2006), por otro lado, (Resende, Batista y Rodrigues, 2008) recomiendan que la construcción de los galpones debe estar orientada de este a oeste para disminuir el impacto directo del sol en los animales por las ventanas laterales de las instalaciones y de esta manera evitar el calentamiento interno del ambiente.

Diversas investigaciones están siendo desarrolladas con el objetivo de buscar mejores resultados y que ayuden a mantener un ambiente térmico ideal para mejorar los rendimientos y establecer condiciones ambientales óptimas a lo largo de toda la producción. De acuerdo con (Abreu y Abreu, 2005) la ventilación es una de las mejores salidas para controlar la temperatura, por otro lado, la temperatura óptima para la producción de pollos de carne oscila entre (29 ${ }^{\circ} \mathrm{C}-32^{\circ} \mathrm{C} ; 26^{\circ} \mathrm{C}-29^{\circ} \mathrm{C} ; 23^{\circ} \mathrm{C}-26^{\circ} \mathrm{C}$ y $20^{\circ} \mathrm{C}-$ $23^{\circ} \mathrm{C}$ ) la segunda, tercera, cuarta y quinta semana respectivamente.

El uso de las Tecnologías de Información y
Comunicación (TIC) pueden ser de gran ayuda para el control térmico en ambientes productivos, asegurando una mayor rentabilidad y bienestar de las aves, por lo que las inserciones de sensores y actuadores en ambientes monitoreados pueden generar informaciones útiles, rápidas y confiables que apoyarían a productores y gerentes en el proceso de toma de decisiones (Corkery, et al., 2013)

\section{MATERIAL Y MÉTODO}

El experimento fue desarrollado en un aviario experimental, en el campus universitario Fernando Costa de la Facultad de Zootecnia e Ingeniería de Alimentos - FZEA de la Universidad de São Paulo USP, en el Municipio de Pirassununga, estado de São Paulo, Brasil situándose entre los meridianos de $21^{\circ}$ 57'37 "S y 47 27'07" W, siendo el clima de Pirassununga, SP; según la clasificación climática de Köppen del tipo Cwa.

El aviario tiene las siguientes dimensiones, 30 metros de longitud por 8 metros de ancho y 4 metros de altura, con cortinas de lona retráctiles climatizada por medios naturales (figura 1), para la obtención de los datos de temperatura se utilizaron ocho sensores (termo - higrómetros DHT22), distribuidos simétricamente dentro del aviario los cuales efectúan mediciones de temperatura de $-40^{\circ} \mathrm{Ca} 125^{\circ} \mathrm{C}$.

Los sensores fueron embarcados en placas microcontroladoras (Figura 2), éstas se conectan a un router Wi-Fi para la transferencia de datos colectados hacia un IP fijo el cual redirige los mismos a una base de datos MySql en la nube (e-Science), siendo el front -end de acceso al sistema e-Science implementado en lenguaje de programación $\mathrm{PhP}$, y acceder en tiempo real vía internet. El experimento fue desarrollado durante el período del 04 de septiembre de 2018 al 01 de octubre de 2018, tiempo durante el cual el aviario permaneció con las cortinas cerradas sin aves en su interior. Los datos procesados son los tomados a las 12 y 15 horas de cada día; estas horas fueron elegidas como muestras porque son las que presentan los mayores picos de temperatura a lo largo del día.

Figura 1. Vista de Aviario, planta baja

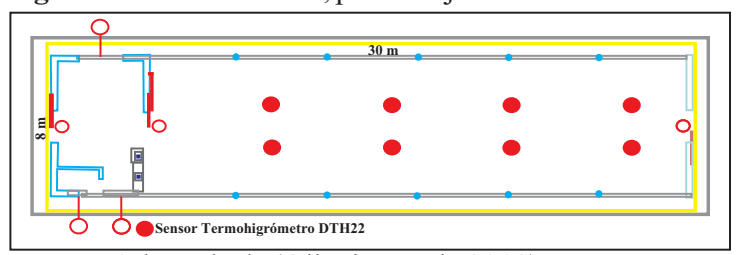

Fuente: Adaptado de (Oliveira, et al., 2018) 
Figura 2: Sensor Termohigrometro DHT22

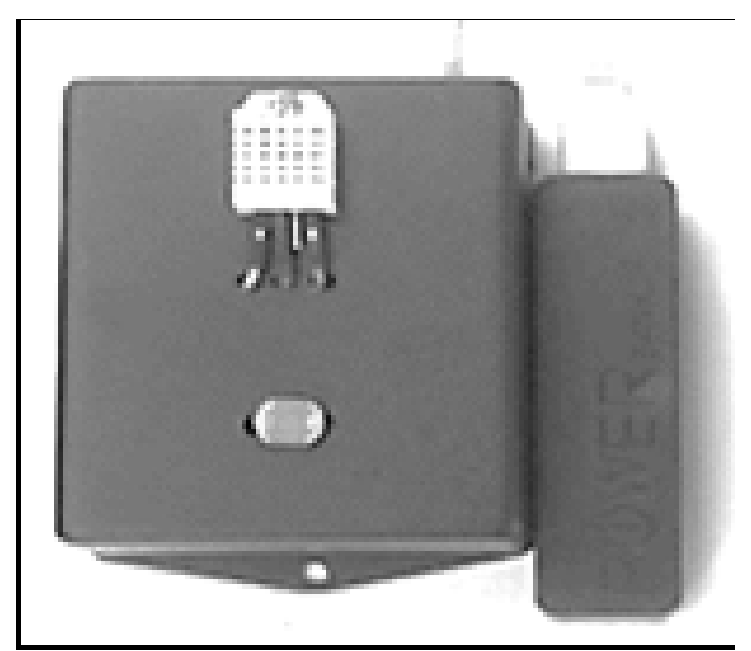

Fuente: Autoría propia

\section{RESULTADOS}

Los datos fueron procesados durante cuatro semanas, asumiendo según Abreu \& Abreu.(2005) pertenecerían a la $2^{\text {da }}, 3^{\text {era }}, 4^{\text {ta }}$ y $5^{\text {ta }}$ semana del ciclo productivo de los pollos de carne, seguidamente se procesaron y evaluaron con la herramienta de control estadístico de proceso (CEP) y el gráfico de media por la amplitud muestral del mismo.

Gráfico 1: Primera semana de experimento, $\mathrm{T}^{\circ}$ de las $12 \mathrm{~h}$.

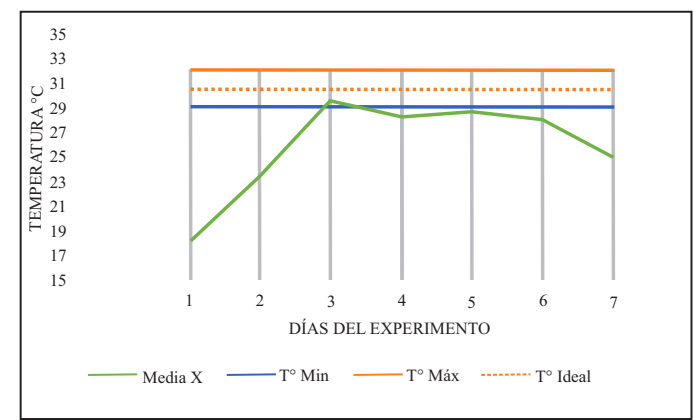

Fuente: Elaboración propia

Gráfico 2: Segunda semana de experimento, $\mathrm{T}^{\circ}$ de las $12 \mathrm{~h}$

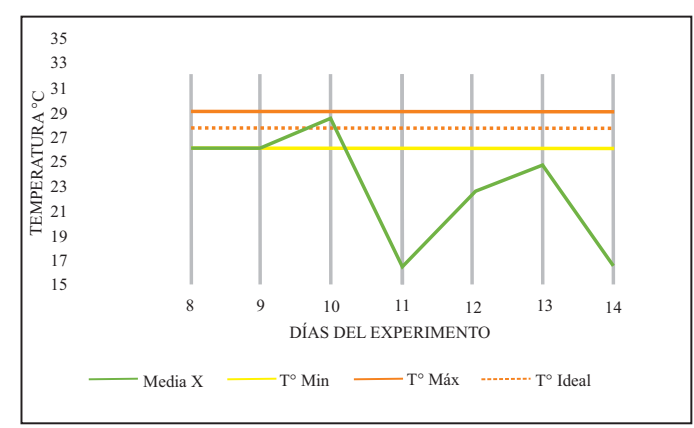

Fuente: Elaboración propia
Gráfico 3 : Tercera semana de experimento, $\mathrm{T}^{\circ}$ de las $12 \mathrm{~h}$.

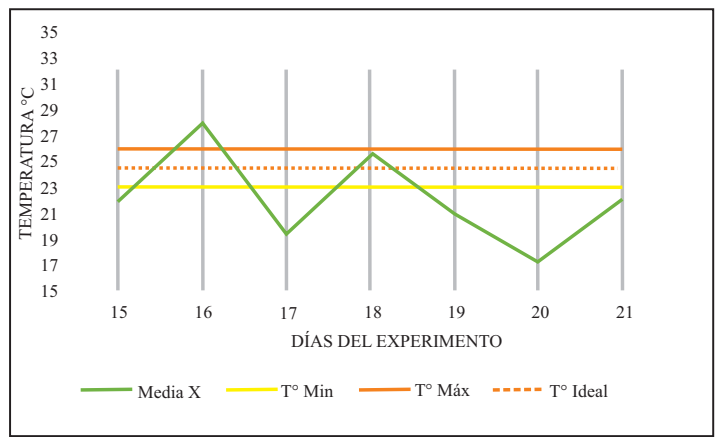

Fuente: Autoría propia

Gráfico 4: Cuarta semana de experimento, $\mathrm{T}^{\circ}$ de las $12 \mathrm{~h}$.

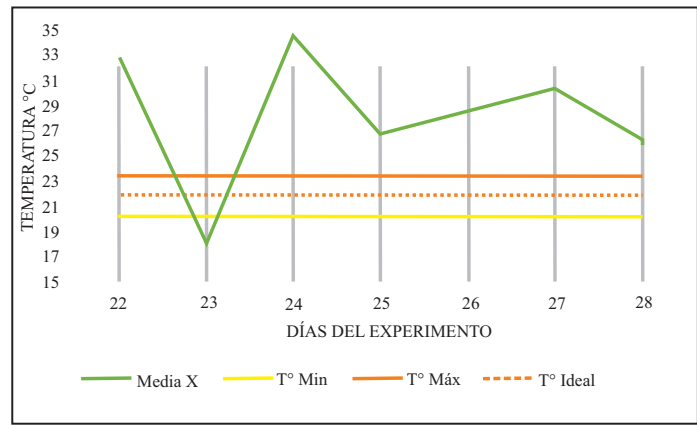

Fuente: Autoría propia

Los gráficos 1, 2, 3 y 4 permiten visualizar el comportamiento de la temperatura tomada de los 8 sensores de las 12 horas de cada día. Se observa una variación entre los límites mínimos y máximos de cada semana establecidos por la literatura.

Gráfico 5: Primera semana de experimento, $\mathrm{T}^{\circ}$ de las $15 \mathrm{~h}$.

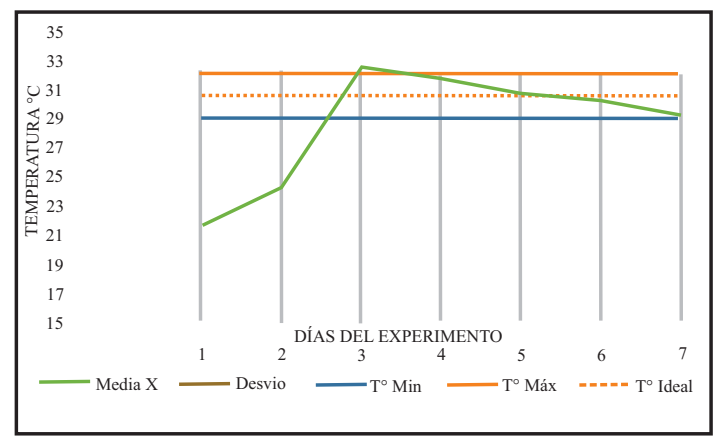

Fuente: Autoría propia 
Gráfico 6: Segunda semana del experimento, $\mathrm{T}^{\circ} \mathrm{de}$ las $15 \mathrm{~h}$.

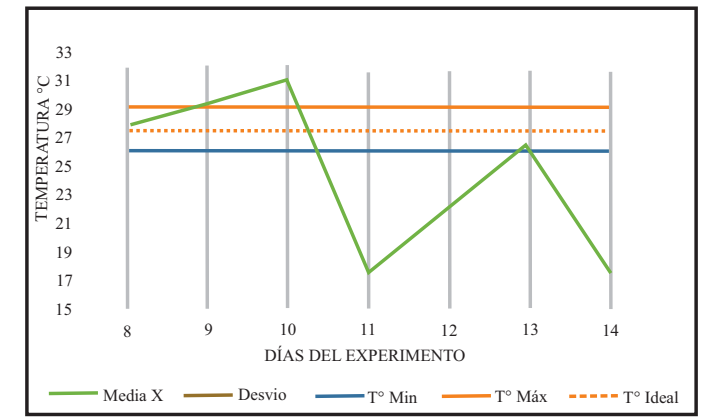

Fuente: Elaboración propia.

Gráfico 7: Tercera semana del experimento, $\mathrm{T}^{\circ} \mathrm{de}$ las $15 \mathrm{~h}$.

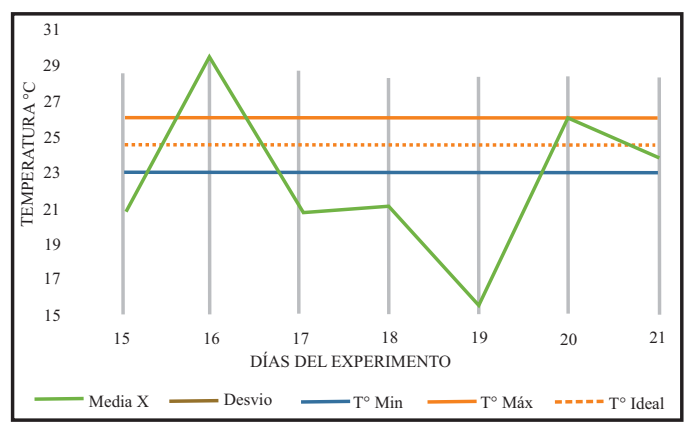

Fuente: Elaboración propia.

Gráfico 8: Cuarta semana del experimento, $\mathrm{T}^{\circ}$ de las $15 \mathrm{~h}$

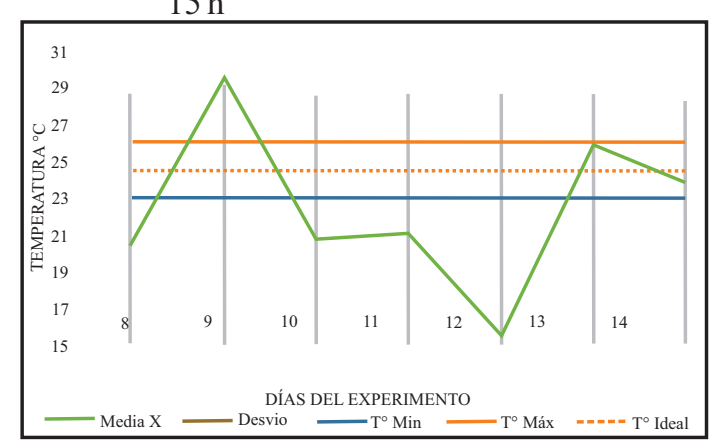

Fuente: Elaboración propia.

Por otro lado, los gráficos 5, 6, 7 y 8 muestran los datos de las temperaturas tomadas de los 8 sensores correspondientes al horario de las 15 horas de cada día. El cual reafirma que el sistema es capaz de proporcionar datos en tiempo real de la temperatura a lo largo del día.

\section{DISCUSION}

Según los gráficos de las temperaturas obtenidas de las 12 horas; se encontraron dentro de la temperatura ideal en cuatro días; 17 días por debajo del mínimo y 7 días por encima del parámetro máximo; con respecto a las temperaturas de las 15:00 horas, en 9 días la temperatura estaba dentro del parámetro ideal, 9 por el debajo del límite mínimo y 10 superior al límite superior; cabe mencionar que durante el experimento se presenciaron precipitaciones durante nueve días lo que explica que los datos obtenidos se encuentran por debajo del parámetro mínimo establecido para cada semana.

En comparación con los comportamientos de las temperaturas de las dos horas analizadas ( 12 y 15$)$, es posible observar mayores intensidades en el período de las 15 horas, esto explica lo que la los índices de temperatura se incrementan a lo largo del día dentro del aviario, esto se explica, por la orientación de construcción del aviario, que fue construido en el sentido norte - sur y, contrario a lo recomendado por Resende, Batista e Rodrigues, (2008), lo que aseguraría una disminución de la incidencia directa del sol en el ambiente.

\section{CONCLUSIONES}

El sistema fue capaz de proporcionar datos de las temperaturas y almacenarlos en la nube MySql, permitiendo así un análisis en tiempo real de las variaciones de temperatura ocurridas en el interior del aviario. Existen procedimientos que pueden ser realizados con el propósito de mantener la temperatura dentro de los límites establecidos por la literatura, para esto el siguiente paso debería ser la implementación de un sistema de actuadores para que al encontrar temperaturas fuera de los parámetros ideales, activen ventiladores, aspersores y cortinas del ambiente a fin de establecer la temperatura y combinación de estos dentro de los parámetros proporcionados por el sistema, evitando así el estrés térmico en las aves y mejorando su bienestar.

\section{REFERENCIAS BIBLIOGRÁFICAS}

Abreu, M.; Abreu, G. Diagnóstico bioclimático para produção de aves na mesorregião Pantanal Sul Mato-Grossense. CONFERÊNCIA APINCO 2005 DE CIÊNCIA E TECNOLOGIA AVÍCOLAS. Santos Anais, Campinas: FACTA. 2005.p. 188.

Abreu, M.; Abreu, G. Os desafios da ambiência sobre os sistemas de aves no Brasil. Revista 
Brasileira de Zootecnia, p. 1-14, 2011.

Abreu, P. G.; Abreu, V. M. Conforto térmico para aves. Concórdia: Embrapa Suínos e Aves, p. 365-366, 2004.

Abreu, V. M.; Abreu, P. G. Diagnostico bioclimático para produção de aves na mesorregião Pantanal Sul Mato-Grossense. Embrapa Suínos e Aves., p. 1-4, 2004.

Bridi, M. Adaptação e aclimatação animal. Universidade Estadual de Londrina, 2010.

Bueno, L.; Rossi, L. A. Comparação entre tecnologias de climatização para criação de frangos quanto a energia, ambiência e produtividade. Revista Brasileira de Engenharia Agrícola e Ambiental, p. $497-$ 504, 2006.

Corkery, G. et al. Monitoring environmental parameters in poultry production facilities. Institute for Process and Particle Engineering, Graz University of Technology, Austria, p. 7-10, 2013.

Fouda, T.; Derbala, A.; Ghoname, M. Some environmental factors affecting broiler housing in winter season. Scientific Papers Series Management, Economic Engineering in Agriculture and Rural Development, p. 2284-7995, 2013.

Han, A. Y. et al. Effect of acute heat stress on calcium concentration, proliferation, cell cycle, and interleukin-2 production in splenic lymphocytes from broiler chickens. Pultry Science, p. 2063-2070, 2010.

Oliveira, M. E. et al. Desenvolvimento de sensores para monitoramento de ambiente aviário com ênfase em controle térmico. Brazilian Journal of Biosystems Engineering, p. 234240, 2018.

Oliveira, R. F. et al. Efeitos da temperatura e da umidade relativa sobre o desempenho e o rendimento de cortes nobres de frangos de corte de 1 a 49 dias de idade. Revista Brasileira de Zootecnia, p. 797-803, 2006.

Resende, O.; Batista, J. A.; Rodrigues, S. Caracterização de instalações avícolas em diversos municípios do estado de Rondônia. Global Science and Technology, p. 1984-3801, 2008.

Silva, A. et al. Avaliação do estresse térmico em condição simulada de transporte de frangos de corte. Revista Brasileira de Zootecnia, p. 1126-1130, 2007. 